\title{
On the Projection onto a Finitely Generated Cone
}

\author{
Miklós Ujvári*
}

\begin{abstract}
In the paper we study the properties of the projection onto a finitely generated cone. We show that this map is made up of finitely many linear parts with a structure resembling the facial structure of the finitely generated cone. An economical (regarding storage) algorithm is also presented for calculating the projection of a fixed vector, based on Lemke's algorithm to solve a linear complementarity problem. Some remarks on the conical inverse (a generalization of the Moore-Penrose generalized inverse) conclude the paper.
\end{abstract}

Keywords: projection map, finitely generated cone

\section{Introduction}

A standard way to generalize concepts in convex analysis is to replace subspaces with polyhedral cones, polyhedral cones with closed convex cones, closed convex cones with closed convex sets, and closed convex sets with closed convex functions. In this paper we make the first step on this way in the case of the concept of the projection onto a subspace, and examine the properties of the projection onto a finitely generated cone. (For higher levels of generality and applications - such as positive linear approximation problems and robotics -, see [3], [5], [6], [12], [13]. For recent results on the projection problem and related algorithms, see [2], [4].)

Let $A$ be an $m$ by $n$ real matrix. Let $\operatorname{Im} A$ resp. Ker $A$ denote the range space (that is the image space) and the null space (that is the kernel) of the matrix $A$. It is well-known that $(\operatorname{Im} A)^{\perp}=\operatorname{Ker}\left(A^{T}\right)$ and $(\operatorname{Ker} A)^{\perp}=\operatorname{Im}\left(A^{T}\right)$ where ${ }^{T}$ denotes transpose and ${ }^{\perp}$ denotes orthogonal complement (see [11]).

The projection map $p_{\operatorname{Im} A}$ onto the subspace $\operatorname{Im} A$ can be defined as follows: for every vector $y \in \mathcal{R}^{m}, p_{\operatorname{Im} A}(y)$ is the unique vector $A x_{*} \in \mathcal{R}^{m}$ such that

$$
\left\|y-A x_{*}\right\|=\min _{x \in \mathcal{R}^{n}}\|y-A x\|, x_{*} \in \mathcal{R}^{n} .
$$

It is well-known that $p_{\operatorname{Im} A}: \mathcal{R}^{m} \rightarrow \mathcal{R}^{m}$ is a linear map: there exists a unique matrix $P_{\operatorname{Im} A} \in \mathcal{R}^{m \times m}$ such that

$$
p_{\operatorname{Im} A}(y)=P_{\operatorname{Im} A} y\left(y \in \mathcal{R}^{m}\right) .
$$

*H-2600 Vác, Szent János utca 1., Hungary. E-mail: ujvarim@cs.elte.hu 
The matrix $P_{\operatorname{Im} A}$ is symmetric (that is $P_{\operatorname{Im} A}^{T}=P_{\operatorname{Im} A}$ ), idempotent (that is $P_{\operatorname{Im} A}^{2}=P_{\operatorname{Im} A}$ ), consequently positive semidefinite (that is $y^{T} P_{\operatorname{Im} A} y \geq 0$ for every $\left.y \in \mathcal{R}^{m}\right)$. Also the equalities

$$
P_{\operatorname{Im} A} \cdot P_{\operatorname{Im} B}=0, P_{\operatorname{Im} A}+P_{\operatorname{Im} B}=I
$$

hold for any matrix $B$ such that $\operatorname{Ker}\left(A^{T}\right)=\operatorname{Im} B$ (see [11]). (Here $I$ denotes the identity matrix.)

Analogous results hold also in the case of the projection onto a finitely generated cone. Before stating the corresponding theorem we fix some further notation.

Let $\mathrm{Im}_{+} A$ resp. $\mathrm{Ker}_{+} A$ denote the so-called finitely generated cone

$$
\operatorname{Im}_{+} A:=\left\{A x \in \mathcal{R}^{m}: 0 \leq x \in \mathcal{R}^{n}\right\}
$$

and the polyhedral cone

$$
\operatorname{Ker}_{+} A:=\left\{x \in \mathcal{R}^{n}: A x \geq 0\right\} .
$$

A reformulation of the Farkas' lemma (see [9], [12] or [13]) claims that $\left(\operatorname{Im}_{+} A\right)^{*}=$ $\operatorname{Ker}_{+}\left(A^{T}\right)$ and $\left(\operatorname{Ker}_{+} A\right)^{*}=\operatorname{Im}_{+}\left(A^{T}\right)$, where $K^{*}$ denotes dual cone (or positive polar) of $K$, that is

$$
K^{*}:=\left\{a \in \mathcal{R}^{d}: a^{T} z \geq 0(z \in K)\right\},
$$

for a convex cone $K \subseteq \mathcal{R}^{d}$. Thus polyhedral cones are the duals of the finitely generated cones, and vice versa. Furthermore, by the Farkas-Weyl-Minkowski theorem (see [9], [12] or [13]), for every matrix $A$ there exists a matrix $B$ such that $\operatorname{Im}_{+} A=\operatorname{Ker}_{+}\left(B^{T}\right)$, or, dually, $\operatorname{Ker}_{+}\left(A^{T}\right)=\operatorname{Im}_{+} B$. In other words, the finitely generated cones are polyhedral cones, and vice versa.

The projection map onto $\operatorname{Im}_{+} A$ can be defined similarly as in the case of $\operatorname{Im} A$ : for $y \in \mathcal{R}^{m}$ let $p_{\mathrm{Im}_{+} A}(y)$ be the unique vector $A x_{*} \in \mathcal{R}^{m}$ such that

$$
\left\|y-A x_{*}\right\|=\min _{0 \leq x \in \mathcal{R}^{n}}\|y-A x\|, 0 \leq x_{*} \in \mathcal{R}^{n} .
$$

For finitely generated cones which are not subspaces this map is not linear anymore, but is made up of linear parts, and these parts have the properties described already in the case of the projection onto a subspace. To state these facts precisely, the definitions of the faces, complementary faces and polyhedral partition are needed.

Let $C$ be a convex set in $\mathcal{R}^{d}$. A convex set $F \subseteq C$ is called an extremal subset (or shortly a face) of the set $C$, if $F$ does not contain an inner point of a line segment from $C$ without containing the endpoints of the line segment, that is

$$
c_{1}, c_{2} \in C, 0<\varepsilon<1, \varepsilon c_{1}+(1-\varepsilon) c_{2} \in F \text { implies } c_{1}, c_{2} \in F \text {. }
$$

We will denote by $F \triangleleft C$ the fact that $F$ is a face of $C$.

If $K$ is the finitely generated cone $\operatorname{Im}_{+} A$, then its faces are finitely generated cones also, and there are only finitely many of them. The faces of (the finitely 
generated cone) $K^{*}$ are exactly the complementary faces $F^{\triangle}$ of the faces $F$ of $K$, defined as

$$
F^{\triangle}:=F^{\perp} \cap\left(K^{*}\right)(F \triangleleft K) .
$$

The complementary face $F^{\triangle}$ can be alternatively defined as

$$
F^{\triangle}=\left\{f_{0}\right\}^{\perp} \cap\left(K^{*}\right)
$$

where $f_{0}$ is an arbitrary element of ri $F$, the relative interior of the face $F$. Also $\left(F^{\triangle}\right)^{\triangle}=F$ holds for every face $F$ of $K$ (see [10] or [13], Theorem 7.27).

It is not difficult to verify using a standard separation argument that if $\mathcal{R}^{d}$ is the finite union of closed convex sets $C_{i}$ with nonempty and pairwise disjoint interiors then the sets $C_{i}$ are necessarily polyhedrons. In this case we call the set $\left\{C_{i}\right\}$ a polyhedral partition of $\mathcal{R}^{d}$.

Now, we can state our main result,

Theorem 1.1. Let $A$ be an $m$ by $n$ real matrix. Let $K$ denote the finitely generated cone $\operatorname{Im}_{+} A$. Then, the following statements hold:

a) The cones $\left\{F-F^{\triangle}: F \triangleleft K\right\}$ form a polyhedral partition of $\mathcal{R}^{m}$. The map $p_{K}$ is linear restricted to the members of this partition, that is for every $F \triangleleft K$ there exists a unique matrix $P_{F} \in \mathcal{R}^{m \times m}$ such that

$$
p_{K}(f-g)=P_{F} \cdot(f-g)\left(f \in F, g \in F^{\triangle}\right) .
$$

b) For every $F \triangleleft K$, and every basis $B$ of $F, P_{F}=P_{\operatorname{Im} B}$. Specially, the matrices $P_{F}(F \triangleleft K)$ are symmetric, idempotent, and positive semidefinite.

c) The map $P$. is a bijection between the sets $\{F: F \triangleleft K\}$ and $\left\{P_{F}: F \triangleleft K\right\}$; and it preserves the usual partial ordering on these sets, that is $F_{1} \subseteq F_{2}$ if and only if $P_{F_{2}}-P_{F_{1}}$ is positive semidefinite.

d) For every face $F$ of $K$,

$$
P_{F} \cdot P_{F}^{*} \Delta=0, P_{F}+P_{F}^{*} \Delta=I .
$$

(Here $P_{F}^{*}$ denotes the matrices defined by $p_{K^{*}}$ obtained via replacing $K$ with $K^{*}$, and $F \triangleleft K$ with $F^{\triangle} \triangleleft K^{*}$ in statement a).)

In Section 2 we will prove Theorem 1.1. In Section 3 we describe an algorithm for calculating the projection $p_{\operatorname{Im}_{+} A}(y)$ for a fixed vector $y \in \mathcal{R}^{m}$. The method is based on Lemke's algorithm to solve a linear complementarity problem LCP (see [1]). After writing the problem as an LCP, using the structure of the problem our algorithm calculates with $r(A)$ by $2 r(A)$ matrices instead of $n$ by $2 n$ matrices $(r(A)$ denotes rank of the matrix $A)$. Finally, in Section 4 we describe a concept closely related to the projection $p_{\operatorname{Im}_{+} A}$ : the conical inverse $A^{<}$. Theoretical and algorithmical properties of the conical inverse are largely unexplored and need further research. 


\section{Proof of the main theorem}

In this section we will prove Theorem 1.1. First we state three lemmas and propositions that will be used in the proof of statement a) in Theorem 1.1.

The first lemma describes a well-known characterization of the projection of a vector onto a closed convex cone, now specialized to the case of a finitely generated cone $K=\operatorname{Im}_{+} A, A \in \mathcal{R}^{m \times n}$ (see [3], Proposition 3.2.3).

Lemma 2.1. For every vector $y \in \mathcal{R}^{m}$ there exists a unique vector $k \in \mathcal{R}^{m}$ such that

$$
k \in K, k-y \in K^{*}, k^{T}(k-y)=0 .
$$

This vector $k$ equals $p_{K}(y)$ then.

As an immediate consequence, we obtain

Proposition 2.1. Let $F$ be a face of $K$. Let $C_{F}$ denote the set of vectors y such that $p_{K}(y) \in$ ri $F$. Then,

$$
C_{F}=(\text { ri } F)-F^{\triangle}
$$

holds.

Proof. Let $C$ denote the set on the right hand side of (2). First, we will show that $C_{F} \subseteq C$. Let $y$ be an element of $C_{F}$, and let $k$ denote the vector $p_{K}(y)$. Then $k \in$ ri $F$; and, by (1), $k-y \in\{k\}^{\perp} \cap K^{*}$, that is $k-y \in F^{\triangle}$. We can see that $y=k-(k-y)$ is an element of $C$, and the inclusion $C_{F} \subseteq C$ is proved.

Conversely, if $k \in \operatorname{ri} F$ and $k-y \in F^{\triangle}$, then (1) holds, so $k=p_{K}(y)$, and we can see that $y \in C_{F}$. This way we have proved the inclusion $C \subseteq C_{F}$ as well.

The closure of the set $C_{F}$ defined in Proposition 2.1 is

$$
\operatorname{cl} C_{F}=F-F^{\triangle} .
$$

The next lemma states that this finitely generated cone is full-dimensional (or equivalently has nonempty interior).

Lemma 2.2. The linear hull of the set $F-F^{\triangle}$ is $\mathcal{R}^{m}$, for every face $F$ of $K$.

Proof. Let $B$ be a matrix such that $K=\operatorname{Ker}_{+}\left(B^{T}\right)$. It is well-known (see [9] or [13], Theorem 7.3) that then there exists a partition $\left(B_{1}, B_{2}\right)$ of the columns of $B$ such that

$$
\begin{aligned}
F & =\left\{y: B_{1}^{T} y \geq 0, B_{2}^{T} y=0\right\}, \\
\operatorname{lin} F & =\left\{y: B_{2}^{T} y=0\right\}, \\
\operatorname{ri} F & =\left\{y: B_{1}^{T} y>0, B_{2}^{T} y=0\right\} .
\end{aligned}
$$

(Here lin denotes linear hull.) Let $f_{0} \in$ ri $F$. Then $F^{\triangle}=\left\{f_{0}\right\}^{\perp} \cap K^{*}$, and the latter set can easily be seen to be equal to $\operatorname{Im}_{+} B_{2}$. Thus the linear hull of $F^{\triangle}$ is $\operatorname{Im} B_{2}$, the orthogonal complement of the linear hull of $F$. The linear hull of the set $F-F^{\triangle}$, being the sum of these two subspaces, is $\mathcal{R}^{m}$. 
It is well-known that relative interiors of the faces of a convex set form a partition of the convex set (see [7], Theorem 18.2): they are pairwise disjoint, and their union is the whole convex set. From this observation easily follows that the sets $C_{F}$ are pairwise disjoint, and their union is the whole space. Consequently their closures, the sets $\mathrm{cl}_{F}(F \triangleleft K)$, have pairwise disjoint interiors (as the interior of $\operatorname{cl} C_{F}$ equals the interior of the convex $C_{F}$ ), cover the whole space, and (by Lemma 2.2) are full-dimensional. We obtained a proof of

Proposition 2.2. The sets $F-F^{\triangle}(F \triangleleft K)$ form a polyhedral partition of $\mathcal{R}^{m}$.

We call a set $C \subseteq \mathcal{R}^{m}$,

- positively homogeneous if $0<\lambda \in \mathcal{R}, y \in C$ implies $\lambda y \in C$;

- additive if $y_{1}, y_{2} \in C$ implies $y_{1}+y_{2} \in C$.

Similarly, we call a map $p: C \rightarrow \mathcal{R}^{m}$, defined on a positively homogeneous, additive set $C$,

- positively homogeneous if $0<\lambda \in \mathcal{R}, y \in C$ implies $p(\lambda y)=\lambda p(y)$;

- additive if $y_{1}, y_{2} \in C$ implies $p\left(y_{1}+y_{2}\right)=p\left(y_{1}\right)+p\left(y_{2}\right)$.

Proposition 2.3. The sets $C_{F}$ defined in Proposition 2.1 are positively homogeneous, additive sets; and the map $p_{K}$ restricted to $C_{F}$ is a positively homogeneous, additive map.

Proof. The first half of the statement follows from Proposition 2.1: the sets (ri $F$ ) $F^{\triangle}$ are obviously positively homogeneous, additive sets.

To prove the remaining part of the statement, let $y \in C_{F}$. Then by Proposition 2.1 there exist $f_{0} \in$ ri $F, g \in F^{\triangle}$ such that $y=f_{0}-g$. Also, for $0<\lambda \in \mathcal{R}$, $\lambda y \in C_{F}$. Again, by Proposition 2.1 there exist $f_{0}(\lambda) \in$ ri $F, g(\lambda) \in F^{\triangle}$ such that $\lambda y=f_{0}(\lambda)-g(\lambda)$. Necessarily,

$$
\lambda p_{K}(y)=\lambda f_{0}=f_{0}(\lambda)=p_{K}(\lambda y),
$$

and we have proved the positive homogeneity of the map $p_{K}$ restricted to the set $C_{F}$. Additivity can be similarly verified, so the proof is finished.

We can see that the sets $C_{F}$ are full-dimensional, positively homogeneous, additive sets, and the map $p_{K}$ restricted to the set $C_{F}$ is a positively homogeneous, additive map. Such maps have a unique linear extension as the following lemma states.

Lemma 2.3. Let $C$ be a positively homogeneous, additive set in $\mathcal{R}^{m}$ such that the linear hull of the set $C$ is the whole space $\mathcal{R}^{m}$. Let $p: C \rightarrow \mathcal{R}^{m}$ be a positively homogeneous, additive map. Then there exists a unique linear map $\ell: \mathcal{R}^{m} \rightarrow \mathcal{R}^{m}$ such that $\ell(y)=p(y)$ for every $y \in C$. 
Proof. Let us choose a basis $\left\{y_{1}, \ldots, y_{m}\right\}$ from the set $C$, and let us define the map $\ell$ as follows:

$$
\ell\left(\sum_{i=1}^{m} \lambda_{i} y_{i}\right):=\sum_{i=1}^{m} \lambda_{i} p\left(y_{i}\right)\left(\lambda_{1}, \ldots, \lambda_{m} \in \mathcal{R}\right) .
$$

We will show that the restriction of this linear map $\ell$ to the set $C$ is the map $p$. Let $C_{0}$ denote the set of the linear combinations of the vectors $y_{1}, \ldots, y_{m}$ with positive coefficients. Then $C_{0}$ is an open set, and $p(y)=\ell(y)$ for every $y \in C_{0}$. Fix $y_{0} \in C_{0}$, and let $y$ be an arbitrary element from the set $C$. Then there exists a constant $0<\varepsilon<1$ such that the vector $y_{\varepsilon}:=\varepsilon y+(1-\varepsilon) y_{0}$ is in the set $C_{0}$. By positive homogeneity and additivity of the map $p$,

$$
p\left(y_{\varepsilon}\right)=\varepsilon p(y)+(1-\varepsilon) p\left(y_{0}\right) .
$$

On the other hand, by linearity of the map $\ell$,

$$
\ell\left(y_{\varepsilon}\right)=\varepsilon \ell(y)+(1-\varepsilon) \ell\left(y_{0}\right) \text {. }
$$

Here $\ell\left(y_{\varepsilon}\right)=p\left(y_{\varepsilon}\right)$ and $\ell\left(y_{0}\right)=p\left(y_{0}\right)$, so we have $\ell(y)=p(y)$; the map $\ell$ meets the requirements.

Finally, uniqueness of the map $\ell$ is trivial, as $\ell$ must have fixed values for the elements of the full-dimensional set $C$.

Now, we can describe the proof of Theorem 1.1.

Proof of part a) in Theorem 1.1: By Proposition 2.3 and Lemma 2.3 existence and uniqueness of matrices $P_{F}$ follow such that

$$
p_{K}(y)=P_{F} y\left(y \in C_{F}\right) .
$$

It is well-known (see Proposition 3.1.3 in [3]), that the map $p_{K}$ is continuous, so we have actually

$$
p_{K}(y)=P_{F} y\left(y \in \operatorname{cl} C_{F}\right) .
$$

We have seen already (see Proposition 2.2) that the sets $\operatorname{cl} C_{F}=F-F^{\triangle}(F \triangleleft K)$ form a polyhedral partition of $\mathcal{R}^{m}$, thus the proof of statement a) in Theorem 1.1 is complete.

Proof of part b) in Theorem 1.1: Let $F$ be a face of the cone $K$. Let $B$ be a basis of the face $F$, and let $B^{\triangle}$ be a basis of the complementary face $F^{\triangle}$. Then every vector $y \in \mathcal{R}^{m}$ can be written in the form

$$
y=B v+B^{\triangle} w, v \in \mathcal{R}^{\operatorname{dim} F}, w \in \mathcal{R}^{\operatorname{dim} F^{\triangle}} .
$$

Multiplying this equality from the left with the matrices $P_{F}$ and $B^{T}$, respectively, we obtain the equalities

$$
P_{F} y=B v, B^{T} y=B^{T} B v
$$


These equalities imply

$$
P_{F} y=B v=B\left(B^{T} B\right)^{-1} B^{T} y=P_{\operatorname{Im} B} y .
$$

We have $P_{F}=P_{\operatorname{Im} B}$, and the proof of statement b) in Theorem 1.1 is finished also.

Proof of part c) in Theorem 1.1: First, notice that the map $P$. is trivially a bijection between the sets $\{F: F \triangleleft K\}$ and $\left\{P_{F}: F \triangleleft K\right\}$. (Injectivity follows from the obvious fact that if $F_{1} \neq F_{2}$, for example there exists $y \in F_{1} \backslash F_{2}$, then $P_{F_{1}}=P_{F_{2}}$ would imply $P_{F_{2}} y=P_{F_{1}} y=y$, and thus $y \in F_{2}$, which is a contradiction.)

Hence, we have to verify only that $F_{1}, F_{2} \triangleleft K, F_{1} \subseteq F_{2}$ implies that $P_{F_{2}}-P_{F_{1}}$ is positive semidefinite. Let $B_{1}$ be a basis of the face $F_{1}$, and let $B_{2}$ be a basis of the face $F_{2}$ such that $B_{1} \subseteq B_{2}$. Then for every $y \in \mathcal{R}^{m}$, by the definition of the projection map,

$$
\left\|y-P_{\operatorname{Im} B_{1}} y\right\|^{2} \geq\left\|y-P_{\operatorname{Im} B_{2}} y\right\|^{2} .
$$

This inequality, by part b) in Theorem 1.1 implies that

$$
y^{T} P_{F_{2}} y \geq y^{T} P_{F_{1}} y\left(y \in \mathcal{R}^{m}\right),
$$

that is the positive semidefiniteness of the matrix $P_{F_{2}}-P_{F_{1}}$, which was to be shown.

Proof of part d) in Theorem 1.1: Let $y_{1}, \ldots, y_{m}$ be a basis in the set $C_{F}$, and let $y_{1}^{\triangle}, \ldots, y_{m}^{\triangle}$ be a basis in the set $C_{F \triangle}^{*}$.

Then, to prove that $P_{F} \cdot P_{F}^{*} \Delta=0$, it is enough to show that

$$
y_{i}^{T} P_{F} \cdot P_{F}^{*} \triangle y_{j}^{\triangle}=0(1 \leq i, j \leq m) .
$$

In other words we have to show that the vectors $p_{K}\left(y_{i}\right)$ and $p_{K^{*}}\left(y_{j}^{\triangle}\right)$ are orthogonal. This follows from the fact that the former vectors are in $F$, while the latter vectors are in $F^{\triangle}$.

To prove the equality $P_{F}+P_{F}^{*} \Delta=I$, it is enough to verify that

$$
y_{i}^{T}\left(P_{F}+P_{F}^{*} \Delta\right) y_{j}^{\triangle}=y_{i}^{T} y_{j}^{\triangle}(1 \leq i, j \leq m) .
$$

In other words that

$$
p_{K}\left(y_{i}\right)^{T} y_{j}^{\triangle}+y_{i}^{T} p_{K^{*}}\left(y_{j}^{\triangle}\right)=y_{i}^{T} y_{j}^{\triangle}(1 \leq i, j \leq m),
$$

or equivalently that

$$
\left(y_{i}-p_{K}\left(y_{i}\right)\right)^{T}\left(y_{j}^{\triangle}-p_{K^{*}}\left(y_{j}^{\triangle}\right)\right)=0(1 \leq i, j \leq m) .
$$

This latter equality is the consequence of the fact that the vectors $p_{K}\left(y_{i}\right)-y_{i}$ are in $F^{\triangle}$ while the vectors $p_{K^{*}}\left(y_{j}^{\triangle}\right)-y_{j}^{\triangle}$ are in $\left(F^{\triangle}\right)^{\triangle}=F$ and so are orthogonal.

This way we have finished the proof of part d), and the proof of Theorem 1.1 as well. 
We conclude this section with an illustrative example. Let us consider the following vectors: $a_{1}:=(1,0)$ and $a_{2}:=(1,1)$, and let $K:=$ cone $\left\{a_{1}, a_{2}\right\}$ (cone denotes convex conical hull). Let $K^{*}$ denote the dual cone of $K$. Then, $K^{*}$ can be described as $K^{*}=$ cone $\left\{a_{1}^{\perp}, a_{2}^{\perp}\right\}$, with $a_{1}^{\perp}=(0,1)$ and $a_{2}^{\perp}=(1,-1)$.

With these notations the faces of $K$ and $K^{*}$ can be given as follows:

$$
\begin{gathered}
F_{0}=\{0\}, F_{1}=\text { cone }\left\{a_{1}\right\}, F_{2}=\text { cone }\left\{a_{2}\right\}, F_{3}=\text { cone }\left\{a_{1}, a_{2}\right\}=K, \\
F_{0}^{\triangle}=\text { cone }\left\{a_{1}^{\perp}, a_{2}^{\perp}\right\}=K^{*}, F_{1}^{\triangle}=\text { cone }\left\{a_{1}^{\perp}\right\}, F_{2}^{\triangle}=\text { cone }\left\{a_{2}^{\perp}\right\}, F_{3}^{\triangle}=\{0\} .
\end{gathered}
$$

By the help of these faces we obtain the following polyhedral partition of $\mathcal{R}^{2}$ :

$$
\begin{gathered}
C_{0}=F_{0}-F_{0}^{\triangle}=-K^{*}, C_{1}=F_{1}-F_{1}^{\triangle}=\text { cone }\left\{a_{1},-a_{1}^{\perp}\right\} \\
C_{2}=F_{2}-F_{2}^{\triangle}=\text { cone }\left\{a_{2},-a_{2}^{\perp}\right\}, C_{3}=K .
\end{gathered}
$$

The projection onto the cone $K$ can be described as follows: for $y=\left(y_{1}, y_{2}\right)$,

$$
p_{K}(y)=\left\{\begin{aligned}
0, & \text { if } y \in C_{0}=-K^{*}, \\
\left(y_{1}, 0\right), & \text { if } y \in C_{1}, \\
\left(y_{1}+y_{2}, y_{1}+y_{2}\right) / 2, & \text { if } y \in C_{2}, \\
y, & \text { if } y \in C_{3}=K .
\end{aligned}\right.
$$

\section{Algorithm for computing the projection}

In this section we will describe an algorithm for calculating the projection of a fixed vector onto a finitely generated cone. The algorithm economically solves a certain type of linear complementarity problems as well.

Let $A$ be an $m$ by $n$ real matrix, and let $K$ denote the finitely generated cone $\operatorname{Im}_{+} A$. Let us fix a vector $y \in \mathcal{R}^{m}$. To compute the projection $p_{K}(y)$, by Lemma 2.1 , we have to find a vector $x \in \mathcal{R}^{n}$ such that

$$
x \geq 0, A x-y \in \operatorname{Ker}_{+}\left(A^{T}\right),(A x)^{T}(A x-y)=0 .
$$

This problem can be rewritten as a linear complementarity problem

$$
L C P(A): \quad\left\{\begin{array}{l}
\text { Find vectors } z, x \in \mathcal{R}^{n} \text { such that } \\
z-A^{T} A x=-A^{T} y ; x, z \geq 0 ; z^{T} x=0 .
\end{array}\right.
$$

A finite version of Lemke's algorithm (see [1]) can be applied to solve $\operatorname{LCP}(A)$; if $(x, z)$ is a solution, then $A x=p_{K}(y)$ is the projection we searched for.

However, a more economical algorithm can be constructed to find the projection of a vector; economical in the sense that instead of solving the $n$-dimensional $\operatorname{LCP}(A)$, it solves a sequence of $r(A)$-dimensional LCPs, $\operatorname{LCP}\left(B_{1}\right), \operatorname{LCP}\left(B_{2}\right), \ldots$, where $B_{1}, B_{2}, \ldots$ are bases of the matrix $A$. (A matrix $B$ is called a basis of the matrix $A$, if $B$ is an $m$ by $r(A)$ submatrix of $A$, and $r(B)=r(A)$.)

Before describing this algorithm, we prove three propositions and lemmas that will be used in the proof of the correctness and finiteness of the algorithm. 
Let $B$ be a basis of the matrix $A$, with corresponding basis tableau $T(B)$. (The basis tableau $T(B)$ corresponding to a basis $B$ contains the unique coefficients $t_{i j} \in \mathcal{R}$ such that

$$
a_{j}=\sum_{i}\left\{t_{i j} a_{i}: a_{i} \in B\right\}\left(a_{j} \in A\right),
$$

where $a_{j}$ denotes the $j$-th column vector of the matrix $A$.) We use the basis tableau $T(B)$ for notational convenience only. In each step of the algorithm we will use only the $j_{*}$-th column of the tableau, that is the solution $t$ of the system $B t=a_{j_{*}}$. Note that $t$ can be calculated using $r(A)$ by $r(A)$ matrices: it is enough to find an $r(A)$ by $r(A)$ invertible submatrix of $B$, and solve the corresponding subsystem of $B t=a_{j_{*}}$.

With these notations,

Proposition 3.1. If $(x, z)$ is a solution of $L C P(B)$, then $B x=p_{\operatorname{Im}_{+} B}(y)$. Furthermore, if

$$
a_{j}^{T}(B x-y) \geq 0\left(a_{j} \in A \backslash B\right)
$$

holds, then $B x=p_{\operatorname{Im}_{+} A}(y)$ as well.

Proof. The statement follows from Lemma 2.1, see also the remark made at the beginning of this section.

If (4) does not hold, then there exist column vectors $a_{j} \in A \backslash B$ such that $a_{j}^{T}(B x-y)<0$. Choose one of them which minimizes the inner product with the vector $B x-y$ : let $j_{*}$ be an index such that

$$
a_{j_{*}}^{T}(B x-y)=\min \left\{a_{j}^{T}(B x-y): a_{j} \in A \backslash B\right\}, a_{j_{*}} \in A \backslash B .
$$

This vector $a_{j_{*}}$ will enter the basis $B$.

From the definition of the index $j_{*}$ immediately follows

Lemma 3.1. The minimum in (5) is less than 0.

Now, we will choose the vector $a_{i^{*}}$ that leaves the basis $B$. Let $i^{*}$ denote an index such that

$$
a_{i^{*}}^{T}(B x-y)=\max \left\{a_{i}^{T}(B x-y): a_{i} \in B, t_{i j_{*}} \neq 0\right\}, a_{i^{*}} \in B, t_{i^{*} j_{*}} \neq 0 .
$$

Remember that $B x=p_{\operatorname{Im}_{+} B}(y)$; so $a_{i}^{T}(B x-y) \geq 0$ holds for every vector $a_{i} \in B$. Hence the maximum in (6) is at least 0 . But more can be claimed:

Lemma 3.2. The maximum in (6) is greater than 0.

Proof. The vector $a_{j_{*}}$ can be written in the form

$$
a_{j_{*}}=\sum\left\{t_{i j_{*}} a_{i}: a_{i} \in B\right\}
$$

If $a_{i}^{T}(B x-y)=0$ would hold for every index $i$ such that $a_{i} \in B, t_{i j_{*}} \neq 0$, then $a_{j_{*}}^{T}(B x-y)=0$ would follow, contradicting Lemma 3.1. 
It is well-known (see [11]) that $t_{i^{*} j_{*}} \neq 0$ implies

Proposition 3.2. The submatrix

$$
\hat{B}:=\left(B \backslash\left\{a_{i^{*}}\right\}\right) \cup\left\{a_{j_{*}}\right\}
$$

is a basis of the matrix A. Furthermore, the corresponding basis tableau $T(\hat{B})$ can be obtained from the basis tableau $T(B)$ by pivoting on the $\left(i^{*}, j_{*}\right)$-th position of the latter tableau.

For the new basis $\hat{B}$ it holds that

Lemma 3.3. The vectors $p_{\operatorname{Im}_{+} B}(y)$ and $a_{j_{*}}$ are elements of the cone $\operatorname{Im}_{+} \hat{B}$.

Proof. As for the solution $(x, z)$ of $L C P(B), z^{T} x=0, z, x \geq 0$ holds, necessarily $x_{i} z_{i}=0$ for all indices $i$. As by Lemma $3.2 z_{i^{*}}=a_{i^{*}}^{T}(B \bar{x}-y)>0$, we have $x_{i^{*}}=0$. We can see that $B x \in \operatorname{Im}_{+}\left(B \backslash\left\{a_{i^{*}}\right\}\right)$; consequently $B x \in \operatorname{Im}_{+} \hat{B}$, that is $p_{\operatorname{Im}_{+} B}(y) \in \operatorname{Im}_{+} \hat{B}$. The remaining statement that $a_{j_{*}} \in \operatorname{Im}_{+} \hat{B}$ is trivial.

The next proposition shows that the new basis $\hat{B}$ is an improvement over $B$.

Proposition 3.3. The distance between $p_{\operatorname{Im}_{+} \hat{B}}(y)$ and $y$ is less than the distance between $p_{\operatorname{Im}_{+} B}(y)$ and $y$.

Proof. Let $S$ denote the following set of vectors:

$$
S:=\left\{s=\varepsilon B x+(1-\varepsilon) a_{j_{*}}: 0<\varepsilon<1, \varepsilon \in \mathcal{R}\right\} .
$$

By Lemma 3.3, $S \subseteq \operatorname{Im}_{+} \hat{B}$. Furthermore, from Lemma 3.1 it can easily be seen that there exists a vector $s \in S$ such that the distance between $y$ and $s$ is less than the distance between $y$ and $B x=p_{\operatorname{Im}_{+} B}(y)$. (In fact, it can easily be verified that

$$
\frac{\|y-s\|^{2}-\|y-B x\|^{2}}{2(1-\varepsilon)} \rightarrow a_{j_{*}}^{T}(B x-y)(\varepsilon \rightarrow 1) .
$$

Applying Lemma 3.1, we have $\|y-s\|<\|y-B x\|$ for some $s \in S$.) As the distance between $y$ and $p_{\operatorname{Im}_{+} \hat{B}}(y)$ is not greater than the distance between $y$ and $s$, so the statement follows.

Now, we can describe the algorithm and prove its correctness.

Theorem 3.1. Algorithm 1 finds $p_{K}(y)$ after finite number of steps.

Proof. The correctness of the algorithm follows from Propositions 3.1 and 3.2. The finiteness of the algorithm is a consequence of Proposition 3.3: as the distance between the vectors $p_{\operatorname{Im}_{+} B}(y)$ and $y$ decreases with each step, so there can be no repetition in the sequence of the bases, and there are only finitely many of the bases of $A$. 


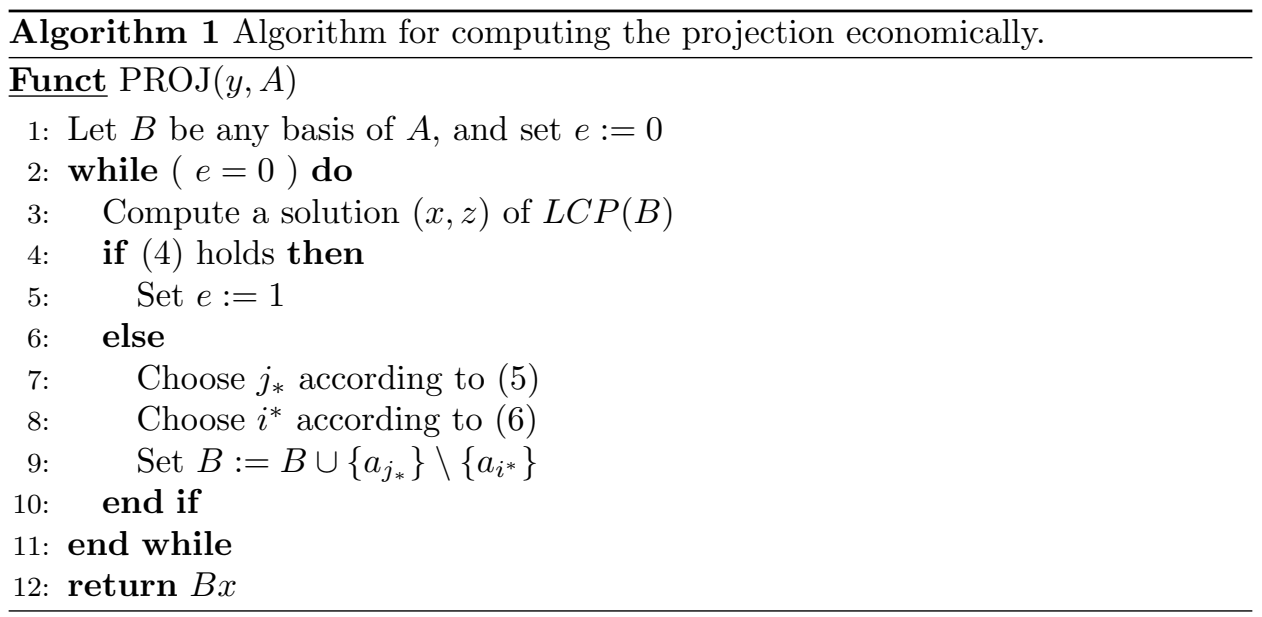

Finally, we remark that Algorithm 1 can be applied to solve economically any LCP of the form

$$
(L C P): \text { Find } x, z \text { such that } z-M x=q ; z, x \geq 0 ; z^{T} x=0,
$$

where $M$ is a symmetric positive semidefinite matrix, $q \in \operatorname{Im} M$. To see this it is enough to note that a matrix $A$ can be found such that $M=A^{T} A$ using Cholesky decomposition (see [11]). Then $\operatorname{Im} M=\operatorname{Im}\left(A^{T}\right)$, so $q=-A^{T} y$ for some vector $y$. This way we have rewritten $(L C P)$ as $L C P(A)$, and the algorithm discussed in this section can be applied to solve the problem.

\section{Remarks on the conical inverse}

In this section we describe a concept closely related to the projection onto a finitely generated cone and also to the Moore-Penrose generalized inverse of a matrix.

Let $A$ be an $m$ by $n$ real matrix. For every vector $y \in \mathcal{R}^{m}$ there exists a unique vector $x_{*} \in \mathcal{R}^{n}$ such that $A x_{*}=p_{\operatorname{Im} A}(y)$ and

$$
\left\|x_{*}\right\|=\min \left\{\|x\|: A x=p_{\operatorname{Im} A}(y), x \in \mathcal{R}^{n}\right\} .
$$

The dependence of the vector $x_{*}$ on the vector $y$ turns out to be linear (see [11]): there exists a unique matrix, called the Moore-Penrose generalized inverse of the matrix $A$ and denoted by $A^{-}$such that $x_{*}=A^{-} y$ for every $y \in \mathcal{R}^{m}$.

It is also well-known (see [8]) that the Moore-Penrose generalized inverse can be alternatively defined as the unique matrix $A^{-} \in \mathcal{R}^{n \times m}$ satisfying the four conditions: a) $A A^{-} A=A$; b) $A^{-} A A^{-}=A^{-}$; c) $\left(A A^{-}\right)^{T}=A A^{-}$; d) $\left(A^{-} A\right)^{T}=A^{-} A$.

Similarly as in the case of the projection map, this concept can also be generalized via replacing the subspace $\operatorname{Im} A$ with the finitely generated cone $\operatorname{Im}_{+} A$. The map defined this way is called the conical inverse of the matrix $A$, and is denoted 
by $A^{<}$. Thus for every vector $y \in \mathcal{R}^{m}, A^{<}(y)$ is the unique vector in $\mathcal{R}^{n}$ satisfying $A A^{<}(y)=p_{\operatorname{Im}_{+} A}(y), A^{<}(y) \geq 0$, and

$$
\left\|A^{<}(y)\right\|=\min \left\{\|x\|: A x=p_{\operatorname{Im}_{+} A}(y), x \geq 0\right\} .
$$

The next proposition describes a certain relation between the two inverses defined above.

Proposition 4.1. For every vector $y \in \mathcal{R}^{m}$, it holds that

$$
(A,-A)^{<}(y)=\left(\max \left\{A^{-} y, 0\right\},-\min \left\{A^{-} y, 0\right\}\right)
$$

where the max and min are meant elementwise.

Proof. Let us consider the following two programs:

$$
(\hat{P}): \quad \text { Find } \min _{x_{1}, x_{2} \geq 0}\left\|y-A x_{1}+A x_{2}\right\|
$$

and

$$
(P): \quad \text { Find } \min _{x}\|y-A x\| \text {. }
$$

The variable transformations

$$
x:=x_{1}-x_{2} \text { resp. } x_{1}:=\max \{x, 0\}, x_{2}:=-\min \{x, 0\}
$$

show the equivalence of programs $(\hat{P})$ and $(P)$.

Furthermore, it can easily be seen that

- if $x$ is an optimal solution of program $(P)$, then the vector

$$
\left(x_{1}, x_{2}\right):=(\max \{x, 0\}+p,-\min \{x, 0\}+p)
$$

is an optimal solution of program $(\hat{P})$ for any vector $p \geq 0$;

- if $\left(x_{1}, x_{2}\right)$ is an optimal solution of program $(\hat{P})$, then the vector $x:=x_{1}-x_{2}$ is an optimal solution of program $(P)$ such that

$$
\left(x_{1}, x_{2}\right)=(\max \{x, 0\}+p,-\min \{x, 0\}+p)
$$

for some vector $p \geq 0$.

Consequently, the optimal solution of $(\hat{P})$ with the least norm will be

$$
\left(x_{1}, x_{2}\right)=(\max \{x, 0\},-\min \{x, 0\}),
$$

where $x$ is the optimal solution of $(P)$ with the least norm; which was to be shown.

Thus any algorithm for calculating the conical inverse can be used for calculating the Moore-Penrose generalized inverse. Conversely also, as the following proposition shows. 
Proposition 4.2. The vector $x$ equals $A^{<}(y)$ if and only if for some vector $z$, $(x, z)$ is a solution of the following linear complementarity problem:

$$
\left(L C P^{<}\right): \quad\left\{\begin{array}{l}
\text { Find } x, z \text { such that } x, z \geq 0 ; z^{T} x=0 \\
x-\left(I-A^{-} A\right) z=A^{-} p_{\operatorname{Im}_{+} A}(y) .
\end{array}\right.
$$

Proof. Let $x_{0} \geq 0$ be a vector such that $A x_{0}=p_{\operatorname{Im}_{+} A}(y)$. To find $A^{<}(y)$ we have to find the unique optimal solution of the following convex quadratic program:

$$
(Q P): \text { Find } \min \left\{\frac{1}{2}\|x\|^{2}: A x=A x_{0}, x \geq 0\right\} .
$$

By the Kuhn-Tucker conditions (see [1]), the vector $x \geq 0$ is an optimal solution of program $(Q P)$ if and only if there exists a vector $z \geq 0$ such that $z^{T} x=0$,

$$
x-z \in \operatorname{Im}\left(A^{T}\right), x-x_{0} \in \operatorname{Ker} A .
$$

It is well-known that

$$
P_{\operatorname{Im}\left(A^{T}\right)}=A^{-} A, P_{\text {Ker } A}=I-A^{-} A,
$$

so (7) can be rewritten as

$$
\left(I-A^{-} A\right)(x-z)=0, A^{-} A x=A^{-} A x_{0} .
$$

It is easy to see that $(8)$ holds if and only if $(x, z)$ satisfies the following equality

$$
x-\left(I-A^{-} A\right) z=A^{-} A x_{0} .
$$

We can see that $x=A^{<}(y)$ if and only if there exists a vector $z$ such that $(x, z)$ is a solution of $\left(L C P^{<}\right)$; the proof is complete.

Note that any problem of minimizing a strictly convex quadratic function

$$
q(x):=\frac{1}{2} x^{T} M x+c^{T} x\left(x \in \mathcal{R}^{n}\right)
$$

(with $M=V^{T} V \in \mathcal{R}^{n \times n}$ symmetric positive definite, $V \in \mathcal{R}^{n \times n}$ invertible, $c \in$ $\mathcal{R}^{n}$ ) over a non-empty polyhedron

$$
\tilde{P}:=\{x: \tilde{A} x=\tilde{b}, x \geq 0\}
$$

(with $\tilde{A} \in \mathcal{R}^{m \times n}, \tilde{b} \in \mathcal{R}^{m}$ ) can be transformed (applying an invertible affine transformation of $\mathcal{R}^{n}$, namely $\left.x:=V x+V^{-1 T} c, x \in \mathcal{R}^{n}\right)$ into the form of $(Q P)$, and in turn $(Q P)$ can be formulated (homogenized) as a projection (of the vector $\left.(0,1) \in \mathcal{R}^{n+1}\right)$ problem onto the polyhedral cone

$$
\left\{\left(\begin{array}{l}
x \\
\lambda
\end{array}\right): A x-\lambda \cdot A x_{0}=0, x \geq 0, \lambda \geq 0\right\} \subseteq \mathcal{R}^{n+1}
$$


(or the constraint $A x=A x_{0}$ can be replaced with $x=x_{0}+B \tilde{x}$, where $B$ is a basis of $\operatorname{Ker} A$, which results again in a projection problem), see [6]. Similarly, the minimizing of the strictly convex quadratic function $q(x)$ over a finitely generated set $\tilde{Q}+\tilde{R}$ (with

$$
\begin{aligned}
\tilde{Q} & :=\left\{\sum_{i=1}^{k} \lambda_{i} \tilde{y}_{i}: \lambda_{i} \geq 0, \sum_{i=1}^{k} \lambda_{i}=1\right\} \\
\tilde{R} & :=\left\{\sum_{j=1}^{\ell} \mu_{j} \tilde{z}_{j}: \mu_{j} \geq 0\right\}
\end{aligned}
$$

for some vectors $\tilde{y}_{i}, \tilde{z}_{j} \in \mathcal{R}^{n}$ ) can be reduced to solving a projection problem onto a finitely generated cone of the form

$$
\left\{\sum_{i=1}^{k} \lambda_{i}\left(\begin{array}{c}
y_{i} \\
1
\end{array}\right)+\sum_{j=1}^{\ell} \mu_{j}\left(\begin{array}{c}
z_{j} \\
0
\end{array}\right): \lambda_{i}, \mu_{j} \geq 0\right\}
$$

for some vectors $y_{i}, z_{j} \in \mathcal{R}^{n}$. The proof of this statement is an adaptation of the results in Chapters 5 and 6 of [6] (as finitely generated sets are polyhedrons, see [7], Theorem 19.1, or [12], [13]), and is left to the reader.

Finally, we mention some open problems concerning the projection and the conical inverse:

- Testing Algorithm 1 on numerical examples (and the comparison of its efficiency with Lemke's algorithm) is a possible direction for further research. What is the number of the smaller LCPs we have to solve in the course of the algorithm?

- Is it true, that similarly to the case of the projection, the conical inverse is also continuous and made up from linear parts? (This statement is trivial if the $m$ by $n$ matrix $A$ has $\operatorname{rank} r(A)=n$.)

- We can see from Proposition 4.2 that the conical inverse for a fixed vector $y$ can be calculated via solving an $n$-dimensional LCP. Is it possible to construct an algorithm to compute $A^{<}(y)$ more economically, similarly as in the case of the projection map? Can this algorithm (or a combination of the two algorithms) be used for solving economically general classes of LCPs?

\section{Conclusion}

In this paper we examined the properties of the projection onto a finitely generated cone. Our main result shows that this map is made up of linear parts with a structure resembling the facial structure of the finitely generated cone we project onto (the map is linear if and only if we project onto a subspace). Also we presented 
an algorithm for computing the projection of a fixed vector. The algorithm is economical in the sense that it calculates with matrices whose size depends on the dimension of the finitely generated cone and not on the number of the generating vectors of the cone. Some remarks and open problems concerning the conical inverse conclude the paper.

\section{Acknowledgments}

I thank János Fülöp and one of the anonymous referees for calling my attention to reference [5], and [2], [4], respectively.

\section{References}

[1] Bazaraa, M. S. and Shetty, C. M. Nonlinear Programming, Theory and Algorithms. John Wiley \& Sons, New York, 1979.

[2] Ekárt, A., Németh, A. B., and Németh, S. Z. Rapid heuristic projection on simplicial cones. Manuscript, 2010.

[3] Hiriart-Urruty, J. and Lemaréchal, C. Convex Analysis and Minimization Algorithms I. Springer-Verlag, Berlin, 1993.

[4] $\mathrm{Hu}, \mathrm{X}$. An exact algorithm for projection onto a polyhedral cone. Australian \&. New Zealand Journal of Statistics, 40: 165-170, 1998.

[5] Lawson, C. L. and Hanson R. J. Solving Least Square Problems. Prentice Hall, Englewood Cliffs NJ, 1974.

[6] Liu, Z. The Nearest Point Problem in a Polyhedral Cone and its Extensions. Phd thesis, North Carolina State University, 2009.

[7] Rockafellar, R. T. Convex Analysis. Princeton University Press, Princeton NJ, 1970.

[8] Rózsa, P. Lineáris Algebra és Alkalmazásai. Tankönyvkiadó, Budapest, 1991.

[9] Schrijver, A. Theory of Linear and Integer Programming. John Wiley \& Sons, New York, 1986.

[10] Stoer, J. and Witzgall, C. Convexity and Optimization in Finite Dimensions I. Springer-Verlag, Berlin, 1970.

[11] Strang, G. Linear Algebra and its Applications. Academic Press, New York, 1980.

[12] Ujvári, M. A Szemidefinit Programozás Alkalmazásai a Kombinatorikus Optimalizálásban. ELTE Eötvös Kiadó, Budapest, 2001. 
[13] Ujvári, M. Konvex Analizis. Manuscript, 2009. URL: http://www.oplab.sztaki.hu/tanszek/letoltes.htm

Received 12th August 2013 